\title{
Algorithm for Brain Tumor Detection
}

\author{
K.Govinda, Dhruv Tiwari, Sachin Mathur, Somula Ramasubbareddy
}

\begin{abstract}
In today's world many engineers have been concentrating in developing various tools for detection of tumor and processing its medical images. The extraction of brain tumor and analysing it is a very challenging task in the field of healthcare. Segmentation's introduction solves the complexity to medical imaging and in turn "MRI (magnetic resonance imaging)" proves to bea very useful diagnostic tool for the detection of brain tumorin MRI's. Here we have performed a comparative study between various clustering and segmentation algorithms. In healthcare field, detection of brain tumor from MRI of the brain, is the current most favourable and seeded area of research. Detecting tumors is one of the major focus areas of the system, it plays a critical role in extraction of details from graphic generated contents of the healthcare. MRI's with brain scans are used in the processes. We have implemented " $k$-means, fuzzy-c means and watershed segmentation"with various soft computing image processing techniques in various test case scenarios which allows us to compare and contrast between the stated techniques. This paper also focuses on enhancing the performance of the algorithms by setting up a suitable parallel environment for these three tumor detection techniques. This will allow multiple MRI's being evaluated simultaneously.
\end{abstract}

\section{INTRODUCTION}

Brain tumor has been a major reason for the increased mortality rate among the adults as well as the children. Tumor is referred as anunusual growth of cells and tissues without any significant use but this growth could be harmless or harmful based on the tumor. Brain tumor is a very serious disease. Many researches have been conducted which showed that the death among people because of brain tumor has greatly increased, speaking in numbers, as much as 300 in the past 3 decades. The most important area and principal regarding medical image processing of tumor and other images of the human body is the pictorial information provided by the processed image. The bio-medical images of the human body are different for different parts of the body. For example, a MRI scan is used for studying the soft tissues of the human body like the brain, liver but for studying hard tissues such as cartilage or bones, $\mathrm{x}$-ray scans are used. These two methods are not only different for different parts of the human body but also differ in the manner in which the image is processed. In other words, we can say that the method used for processing X-ray images is very different from the method

used for processing MRI scanned images. Tumor in the brain is a specific portion, where an outgrowth is seen. Brain scans

Revised Manuscript Received on September 22, 2019

K.Govinda, Associate Professor, School of Computer Engineering,VIT-Vellore.

Dhruv Tiwari, Computer Science and Engineering (Bioinformatics), VIT-Vellore

Sachin Mathur, Computer Science and Engineering (Bioinformatics), VIT-Vellore

Somula Ramasubbareddy, Assistant Professor, Information Technology, VNR VJIET, Hyderabad, Telangana.

for the brain tumor can be done by, "Computer tomography (CT) scan, magnetic resonance image (MRI) scan, Ultrasound", etc. In our implementations, MRI'sare utilised. Variousmethodshave been seen for detection of the brain tumorlike a method using connected component analysis. The method presented by Lo Hall required image cropping for the exact result of the tumor region but is not obtained. Walters suggested using K-means Clustering. Kaur presented using soft computing method. Therefore the process would cause faulted detection in observing the scans. In our experiment we have used and reviewed "K-means clustering", "Fuzzy-c means" and "watershed segmentation" techniques to detect brain tumor. The k-means clustering is done on brain-mri images for clustering which in turn segregates the tumor region as cluster in whole. Whereas fuzzy-c means allows multiple points to lie in multiple clusters thus allows working with clusters with more details.The tumor region gets classified into the tumor category, while the rest are inserted into the non-tumor category. This will be the serial execution of the code, to make the more much efficient we have introduced the concept of parallelization. Suppose the user wants to detect tumor regions from various perspective or he want to detect tumor in more than one MRIs using the proposed concept then the user will have to go through the whole code individually of individual MRIs thus will consume a lot of time as the serial execution of the code in itself is very computationally intensive, thus by introducing parallelism in the code we have drastically reduced the time required for the computation of more than one MRIs thereby not only making the algorithms handle load easily but also very efficient.

\section{BRAIN TUMOR DETECTION}

Prof. Dinesh D. Patil(2013), has expressed the outline of different segmentation approach implemented for medical imaging has been briefed. The paper likewise goes around the examination on different research techniques connected for image segmentation and different research issues in this field. This research expects to give a basic manual for the scientist for those performed their exploration contemplate in the image segmentation. Image segmentation has a promising future as the general division calculation and it has turned into the focal point of contemporary research. Notwithstanding a very long while of research up to now to the learning of creators, there is no all-around acknowledged technique for image segmentation, as the consequence of segmentation is influenced by bunches of elements, for example, homogeneity of pictures, spatial attributes of the picture progression, surface, picture content. 
In this way there is no single technique which can be viewed as useful for neither all kind of pictures nor all strategies similarly useful for a specific sort of picture. Because of every above factor, medical image segmentation remains a difficult issue in picture handling and $\mathrm{PC}$ vision is as yet a pending issue in the world of healthcare.

Maoguo Gong (2013), exhibited an enhanced fluffy C-means (FCM) calculation for image division by presenting a tradeoff weighted fluffy factor and a kernel metric. The tradeoff weighted fluffy factor relies upon the space separation of every neighbouring pixel and their gray level contrast at the same time. The new calculation adaptively decided the portion parameter by utilizing a quick transmission capacity choice manage dependent on the separation change of all information focuses in the accumulation. Moreover, the tradeoff weighted fluffy factor and the portion remove measure are both parameter free. Trial results on engineered and genuine pictures demonstrate that the new calculation is powerful and productive, and is generally free of this sort of noise.

Bhagwat et al (2013) they demonstrated that DICOM pictures create better outcomes when contrasted with non medical pictures. They found that time prerequisite of hierarchical clustering was minimum of three and that for Fuzzy C means it was most elevated for recognition of cerebrum tumor. K-means calculation delivers more precise outcome contrasted with Fuzzy c-means and hierarchical clustering.13]

A.Sivaramakrishnan and Dr.M.Karnan(2013) proposed a novel and an effective recognition of the brain tumor district from cerebral picture was finished utilizing Fuzzy C-means clustering and histogram. The histogram adjustment was utilized to compute the power estimations of the dark level pictures. The decomposition of pictures was finished utilizing guideline part examination which was utilized to lessen dimensionality of the wavelet co - effective. The aftereffects of the proposed Fuzzy C-means (FCM) clustering calculation effectively and precisely separated the tumor district from cerebrum MRI mind images[11]

Jaskirat kaur et al (2012), depicted clustering calculations for picture segmentation and completed a survey on various types of picture division techniques. They likewise proposed a system to order and measure diverse grouping calculations dependent on their consistency in various applications. They portrayed the different execution parameters on which consistency will be estimated.

Roy et al (2012) computed the tumor influenced region for symmetrical investigation. They demonstrated its application with a few informational indexes with various tumor size, force and area. They demonstrated that their algorithm can naturally identify and section the brain tumor. MR pictures gives better outcome contrast with different systems like CT pictures and X-rays. Picture pre-processing incorporates change of RGB picture into grayscale picture and afterward passing that picture to the high pass filter with the end goal to expel noise present in image.[14]

B. Sathya et al (2011), proposed four clustering algorithm; $\mathrm{k}$ mean, enhanced $\mathrm{k}$ mean, $\mathrm{c}$ mean and enhanced $\mathrm{c}$ mean calculation. They completed a test investigation for huge database comprising of different pictures. They dissected the outcomes utilizing different parameters.
Hui Zhang et al (2008), thought about abstract and administered evaluation approach for image division. Subjective assessment and supervised assessment, are infeasible in numerous vision applications, so unsupervised strategies are important. Unsupervised assessment empowers the target examination of both distinctive division techniques and diverse parameterizations of a solitary method.[6]

Military Heber et al (2005), exhibited an evaluation of two prevalent segmentation algorithms, the mean move based division calculation and a chart based division conspire.

Padmakant Dhage(2015),presented a research where watershed has been a proactive techniques to detect brain tumors, they specified why watershed is a good technique to be implemented using MATLAB as it can be used to segment the MRI images quite efficiently.

Priyanka Shah(2017), reviewed and compared multiple clustering and segmentation techniques and suggested why watershed segmentation is a better and an accurate algorithm and they have shown how and why which algorithm lacks behind in certain aspects.

\section{ALGORITHMS WORKED UPON:}

\section{Fuzzy C means}

FCM (fuzzy c means) can be considered a modern technique used for clustering allowing the single data point be into clusters, thus using the fuzzy algorithm creating certain fuzzy sets in which a data point also lies into various other fuzzy sets. This technique is known and has proven successful for pattern matching and recognition. The algorithm assigns partial values of pixel to each data point and hence allowing them to be in multiple fuzzy clusters. The fuzzy member values are calculated and assigned using the below function and also lies under the algorithm for the same.

$$
\mathrm{u}_{\mathrm{ij}}=\frac{1}{\sum_{k-1} c\left(\frac{\| x i-c j \mid}{\| x i-c j||}\right) 2 / m-1}
$$

\begin{tabular}{|c|}
\hline $\begin{array}{l}\text { Step 1: } \quad \text { Initialize } \mathrm{U}=\left[\mathrm{u}_{\mathrm{ij}}\right] \text { matrix, } \mathrm{U}^{(0)} \\
\text { Step2: At k-step: calculate the centers } \\
\text { vectors } \mathrm{C}^{(\mathrm{k})}=\left[\mathrm{c}_{\mathrm{j}}\right] \text { with } \mathrm{U}^{(\mathrm{k})} \\
\qquad \mathrm{c}_{\mathrm{j}}=\frac{\sum_{i-1}^{N} \mathrm{u}_{i j}^{m}, x i}{\sum_{i-1}^{N} \cup_{i j}^{m}}\end{array}$ \\
\hline $\begin{array}{l}\text { Step:3 Update } \mathrm{U}^{(\mathrm{k})}, \mathrm{U}^{(\mathbf{k}+1)} \\
\quad \mathrm{u}_{\mathrm{ij} j}-\frac{1}{\left.\sum_{k-1}^{c}\left(\frac{\| x i-c j \mid}{\| x i-c j \mid}\right)\right)^{2 / m-1}}\end{array}$ \\
\hline $\begin{array}{l}\text { Step4: If }\left\|\mathrm{U}^{(\mathrm{k}+1)}-\mathrm{U}^{(\mathrm{k})}\right\|<\varepsilon \text { then STOP; } \\
\text { otherwise return to step } 2 \text {. }\end{array}$ \\
\hline
\end{tabular}

Figure 1. Fuzzy Member Function 
The steps included in the algorithm are as follows:

1. Select an image form the database.

2. Check whether the image is in grayscale, if not convert it into grayscale.

3. Covert the image into Double.

4. Predefine the number of clusters and iterations

5. Convert the matrix of input image into a vector

6. Select the k-cluster centre randomly.

7. Calculate the fuzzy centre using

$$
R_{i}-\sum_{i=1}^{n} x n_{i} M \eta l \sum_{i=1}^{n} M \eta
$$

8. Using the distance formula, calculate the fuzzy membership function.

$$
\mathcal{M}_{i}=\frac{1}{\sum_{i=1}^{n}\left(\left\|\frac{x_{i}-x_{i}}{x_{i}-c_{i}}\right\|\right)^{\frac{2}{n-1}}}
$$

Repeat the steps 7 and 8 until a minimum value is achieved

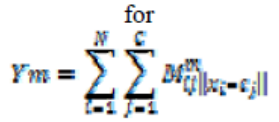

Algorithm 1. Fuzzy-C Means

\section{K Means:}

$\mathrm{Km}$ (K means algorithm) can be considered as the most basic learning algorithm that is unsupervised in nature and is used to take care of notable issue of grouping. This method pursues simple and very basic idea for a provided or known informational index characterization via bunches that are fixed in number (k groups) fixed beforehand. Principle process can be specified as the $\mathrm{k}$ focuses characterization, one belongs each group. On various different areas causing distinctive outcomes accounts these focuses are supposed to be put shrewdly. It is always preferred or considered better to put them as far as expected from each other along the lines(these lines). The stage that follows is partnering of each point to the focus that is closest and that is having a place towards or to a known informational index. When pending status of all points are over, marks the end of initial step and thus finished early age of cluster. Re-computation of $k$ new centroids as barycentre of the clusters that came to be because of previous steps or the steps before, is the next step. In the next stepagain coupling is to be done between closest focus that are new and similar centres, after the availabilityk new centroids. Creation of a circle comes into the scene. Due to this circle changes in the $\mathrm{k}$ focuses area that are well ordered until no changes takes place anymore is noticed. At last, this calculation proceeds for limiting a target work called squared error function which can also be explained as:

$$
J(V)=\sum_{i=1}^{c} \sum_{j=1}^{c_{i}}\left(\left\|x_{i}-v_{j}\right\|\right)^{2}
$$

where,

$$
\begin{aligned}
& \text { ' }|| x_{i}-v_{j}|| \text { ' is the Euclidean distance between } x_{i} \text { and } v_{j} \\
& \text { ' } c_{i} \text { ' is the number of data points in } i^{t h} \text { cluster. } \\
& \text { ' } c \text { ' is the number of cluster centers. }
\end{aligned}
$$

Figure 2. Squared Error Function

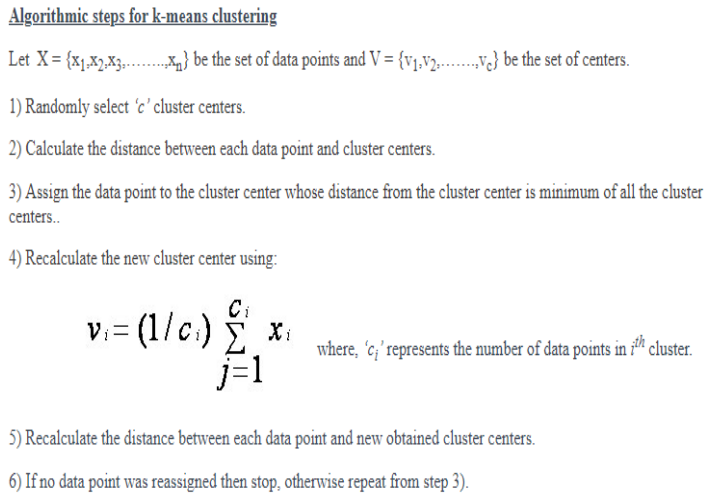

Algorithm 2. K-Means

\section{Watershed Segmentaion:}

The watershed segmentation uses the pixel map to generate a gradient map and then the technique is used for image segmentation and object separation.Representing a 2D image section in the form of 3D with the help of valleys and hills in the area of the working. This technique can be used efficiently with the suggested method in the paper, as the unique feature of the gradient can be used to get extra detail about the tumor.

The steps involved in this algorithm are:

1. Read the input image.

2. Convert input image into grayscale image.

3. Compute foreground markers(connected blobs and pixels).

4. Compute background markers.

5. Compute the Watershed transform of the segmentation function.

6. Output image.

\section{MODEL DIAGRAM}

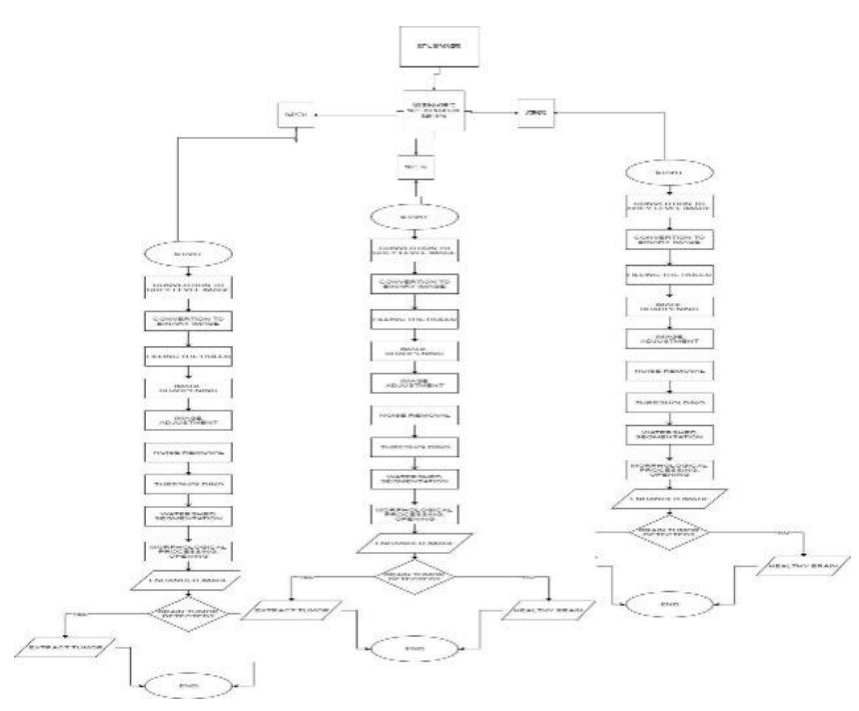

Figure 3. Model Diagram for Parallel Execution 


\section{SUGGESTED METHODOLOGY}

Here, we have proposed a method that not only increases the efficiency and accuracy of watershed segmentation but also enables us to extract tumor sections from the MRI of the brain without using any time consuming and compute intensive algorithm by using pre-processing and morphological operations combined with watershed segmentation,

\section{Text removal}

Covert the image to gray level. Thenconvertit to
binary image.
After that fill the holes. Use
ExtractLargestBlobs.

\section{Make Enhancement for the image}

In this step we are making enhancement for the received image from previous step withthe following methods:

* Sharpening: A version of the grayscale image that is enhancedwhere the features of image like edges are sharpened.

* Image adjust: increase the contrast (separation between the dark and white colors) of the image and saturate the high and low intensities.

\section{Applying noise removal filters}

In this step we try many Image processing filters like min, max, median and mean filters and the gray scale image obtained so as to obtain noiseless image.

\section{Make thresholding and segmentation}

This step concerns with image analysis technique segmentation portioning the image into a foreground and background, so we can isolate the needed object by converting the image into black and white (binary image).

\section{Watershed Segmentation}

This technique is used for image segmentation and object separation.Representing a 2D image section in the form of $3 \mathrm{D}$ with the help of valleys and hills in the area of the working.

\section{Morphological operations}

In this steps we will use opening which is consists of two operation erosion followed by dilation we selected the opening operation as it is spot and noise removal and less destructive, and after that apply Gaussian filter to smooth the final image.

\section{PARALLEL EXECUTION}

NOTE: In this project we have utilized the PARALLEL COMPUTING TOOLBOX i.e. an extension of matlab for the parallel execution. This toolbox assigns workers or cores available and they work in parallel. Thus parts specified under the parfor loop (a command to execute for loop in parallel with the help of the workers assigned to the code) is executed in parallel.

\section{Identification of areas that can be executed in Parallel}

Before this module morphological operations marks the end of the serial execution of the code i.e. the one time working of the code with the single MRI image available. Now in this module, the execution of this code is taken to area that requires more than 1 execution of MRI or execution of MRI from various angels etc. Single Serial execution being computationally intensive in itself makes the execution of more than one MRIs even more time consuming. Thus various regions in the code is identified that can be executed in parallel so as reduce the time taken by single execution of the code.

\section{Applying the parallel execution.}

After the identification of regions in which parallel execution can be applied i.e.

\section{Executing the whole code in parallel as a function:}

This is the other and more efficient version of execution. Parallel computing toolbox allows two functions to be executed in parallel only and only if there is no connection between the two functions i.e. neither is dependent on the other in any case. Thus if we see, the whole code i.e. one time execution is complete in itself thus if the code is used to create functions then the functions will have no connection with each other thus neither is dependent on the other thus can be executed in parallel. Now setting different MRIimage as input for each function we can execute or compute or work with more than one MRIs at the same time in parallel, thus reducing the computation time of more than one MRIs drastically.

\section{V.EXPERIMENT DISCUSSION:}

The experiment that we have performed here for the purpose of brain tumor detection includes fuzzy $\mathrm{c}$ means algorithm, K-means algorithm, and Watershed Algorithm used along with various soft computing techniques. Each of the algorithm is examined and the time required by each of the algorithm along with the accuracy of each taken from the experiment as well as the published works ${ }^{[18][19]}$, also we have experimented with combination of algorithms i.e. fuzzy c means followed by watershed segmentation. Thereby we tried to suggest which algorithm will work the best in which conditions.

We have also created a parallel environment for the execution of all these algorithms thereby allowing the use of increased resources for decreasing the time in case of multiple input images for all the algorithms. The environment is created in such a way that matlab assigns workers that will inturn handle section of code assigned to it in parallel. Here each algorithms will be used to create 
an isolate functions, then these functions are provided with different inputs and thereby with the PARFOR loop which demands independent functions, these algorithms are executed on more than one image at a time in parallel.

\section{Fuzzy C-means:}

Since this algorithms tries to assign each point to more than one cluster thus is very complex and time consuming but also accurate. Here we noticed that the quality of the image changed the time taken for the clustering to increase drastically as the number of pixels present in an image to work with increases drastically with the increasing quality of the image. In sequential execution of the code the time taken for the normal quality brain MRI was very low and since the computation was not that intensive the time difference when running 2 images in sequential as well as parallel was almost absent. But as the input image quality increased the time and it can be explained in this way to that increasing the quality increases pixels and fuzzy c means assigns each pixel to more than one cluster at a time thereby increasing its complexity drastically. Now the problem is very intensive thus significant difference was noted when 2 high quality MRIs were executed in sequential as well as when executed in parallel.

Outputs of both the sequential as well as parallel are

\section{Sequential:}

\section{Normal Quality}
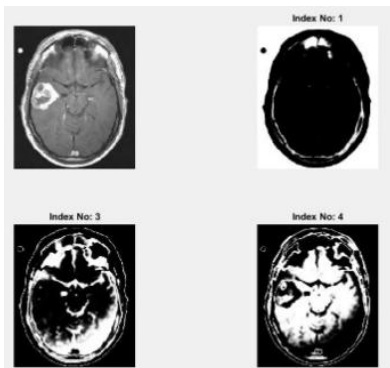

Figure 4. Output of Fuzzy-C means normal quality in sequential

\section{High Quality:}

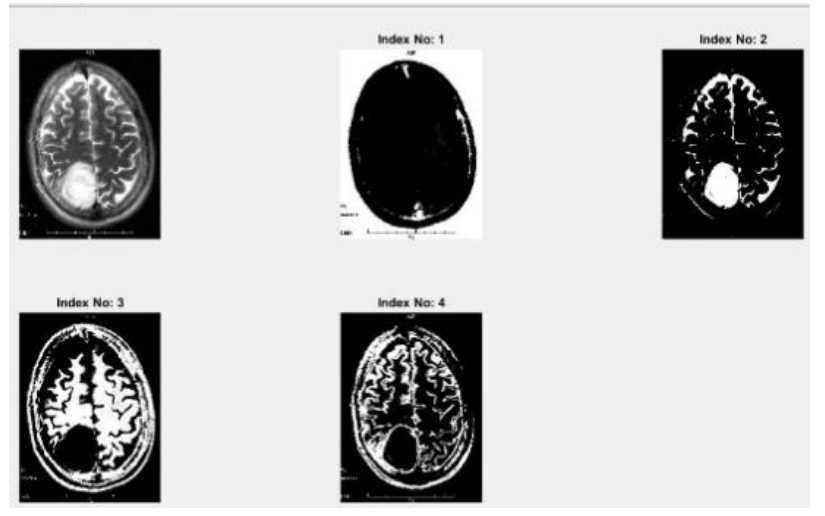

Figure 5. Output of Fuzzy-C means High Quality required for the sequential execution increased drastically followed:

\section{Parallel (2 images simultaneously) :}
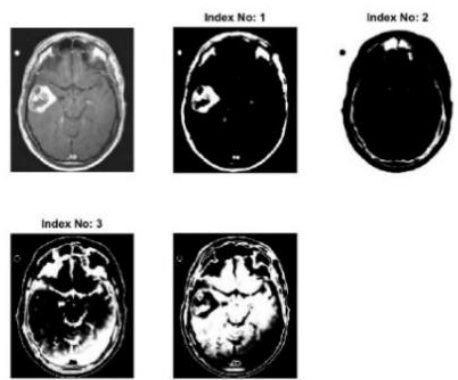

Figure 6. Output of Image 1 in parallel for Fuzzy-C means
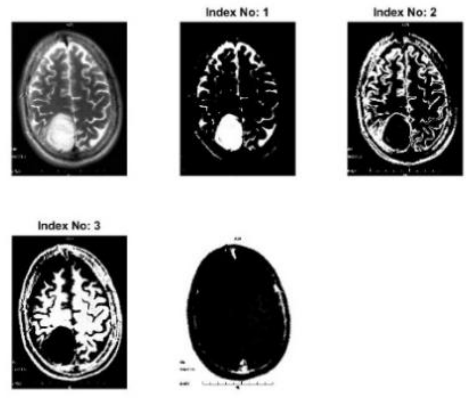

Figure 7. Output of Image 2 in parallel for Fuzzy-C means

\section{K Means:}

This algorithm as not as computationally intensive as the above algorithms thus there was almost no effect of the image's quality was noted in this algorithm. Since in this algorithm each pixel only belong to one cluster at a time. The algorithm is able to segment the tumor region properly as that can be seen from the images below. Parallel execution of the code also witnessed a significant decrease in time required for executing 2 images in sequential as well as executing 2 images in parallel with this algorithm.

NOTE: For this algorithm we have not implemented any high quality image separately because time difference between $\mathrm{K}$ means and Fuzzy C-means is coming out to be very less as $\mathrm{K}$ means take 3.170 s for a image size and fcm takes $4 \mathrm{~s}$ for the same size image and fuzzy c means though takes more time but is much more accurate and efficient as compared to $\mathrm{K}$ means because of its property of assigning each data point to all the clusters as compared to $\mathrm{k}$ means which allows a data point to be a part of one cluster only thus if the wrong initial cluster is chosen chances of wrong output is there which is completely removed in case of fcm due to its optimal cluster centre selection.

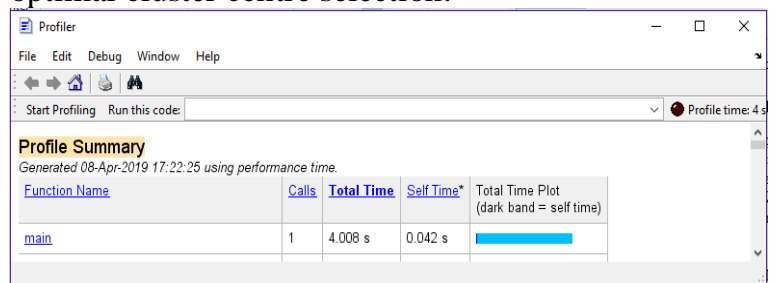

Figure 8. Time of Fuzzy c-means when executed for same image size as in K-means 
Sequential:
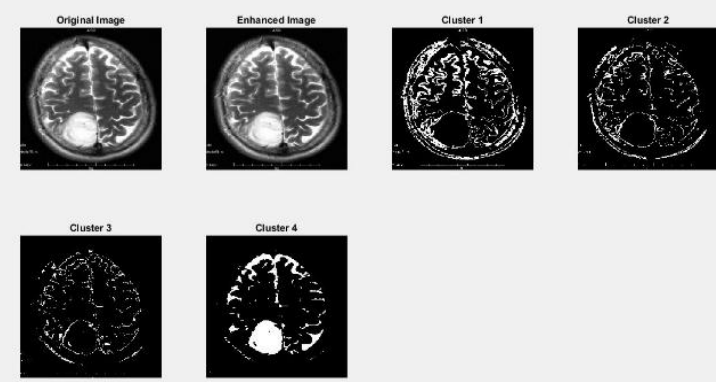

Figure 9. Output of K-means in sequential

Parallel (2 images simultaneously):
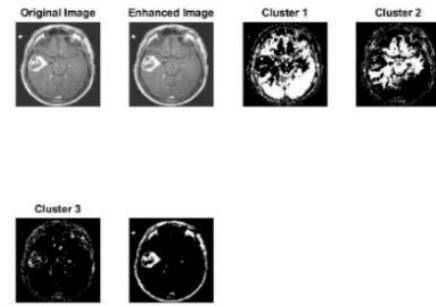

Figure 10. Output of Image 1 in parallel for K-means
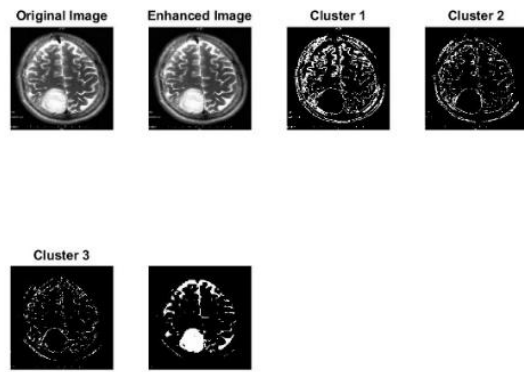

Figure 11. Output of Image 2 in parallel for K-means

Watershed Segmentation with various soft computing image techniques for enhancement of image (suggested):

Pre-processing used with watershed segmentation on the MRI image is one of the main important techniquethat smooth image and does noise removal. Stripping of skull and removal of text is done to avoid misclassification of non-brain and brain tissues.Following this, enhancement of image and thresholding is done. After this, with the help of marker-controlled Watershed segmentation image segmentation is done.From the image that is segmented the region having tumour is located with morphological operations and the tumour region that is calculated can be visualized.

Thus here instead of using clustering algorithms for the detection of the tumor section, various soft computing image processing techniques are used i.e. pre-processing thereby highlighting the tumor section properly thereby allowing the proper application of watershed segmentation. This technique is very much time efficient as compared to other clustering algorithms as not clusters are created instead the tumor section is highlighted with the help of image processing techniques, and thus application of watershed segmentation is done properly thus somewhat increasing the accuracy of the algorithm as well. Thus with watershed we can have $3 \mathrm{~d}$ representation of $2 \mathrm{~d}$ MRIs.

\section{Sequential:}
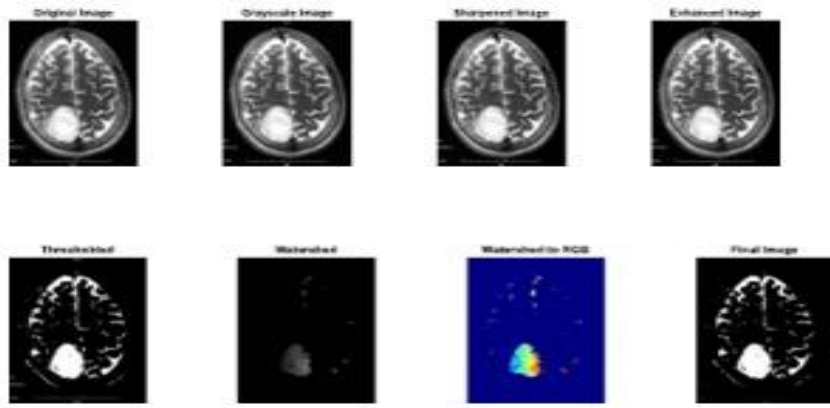

Figure 12. Output of Watershed Segmentation in sequential

Parallel (2 images simultaneously):
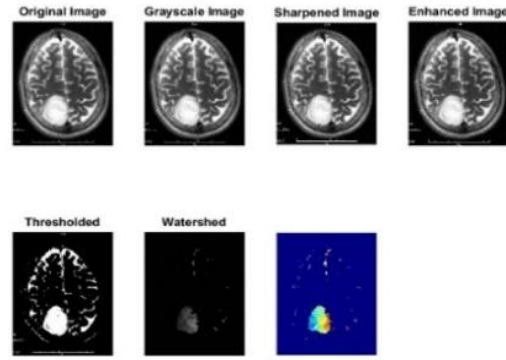

Figure 13. Output of Image 1 in parallel for Watershed Segmentation
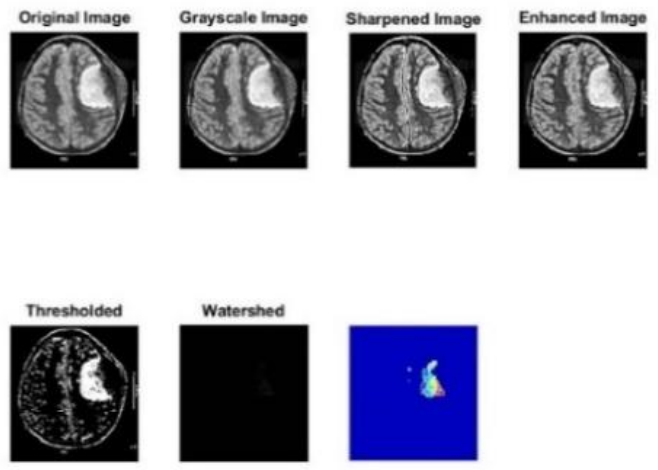

Figure 14. Output of Image 2 in parallel for Watershed segmentation

\section{Fuzzy C Means with Watershed Algorithm:}

We have also testing the combination of Fuzzing C Means with Watershed Algorithm, since the accuracy of FCM is very high thus using this technique increases the accuracy of watershed as well but the time taken for execution of MRIs also increases drastically and that is a trade-off when using this techniques i.e. High time with very high accuracy.

Published By:

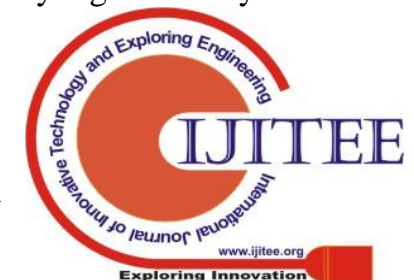




\section{EXPERIMENTAL RESULTS:}

\begin{tabular}{|l|c|c|c|}
\hline Algorithms & $\begin{array}{c}\text { Sequential Time Taken } \\
\text { (Time taken for } 1 \text { MRI Image) }\end{array}$ & $\begin{array}{c}\text { Parallel Time Taken } \\
\text { (Time taken for 2 MRI } \\
\text { Image in parallel) }\end{array}$ & $\begin{array}{c}\text { Time Taken With High } \\
\text { Resolution Image }\end{array}$ \\
\hline K-means & 3.170 seconds & 3.372 seconds & $\begin{array}{c}\text { Not much difference was } \\
\text { observed in the } \\
\text { performance }\end{array}$ \\
\hline $\begin{array}{l}\text { Fuzzy-C } \\
\text { Means }\end{array}$ & 3.675 seconds & $\begin{array}{c}6.743 \\
\text { seconds//71.452(HQ) }\end{array}$ & $\begin{array}{c}39.158 \text { seconds } \\
\text { Huge performance } \\
\text { difference was observed }\end{array}$ \\
\hline $\begin{array}{l}\text { Watershed } \\
\text { Segmentation }\end{array}$ & 1.693 seconds & 1.968 seconds & $\begin{array}{c}\text { No evident difference in } \\
\text { performance }\end{array}$ \\
\hline
\end{tabular}

Table 1. Execution time for the algorithms

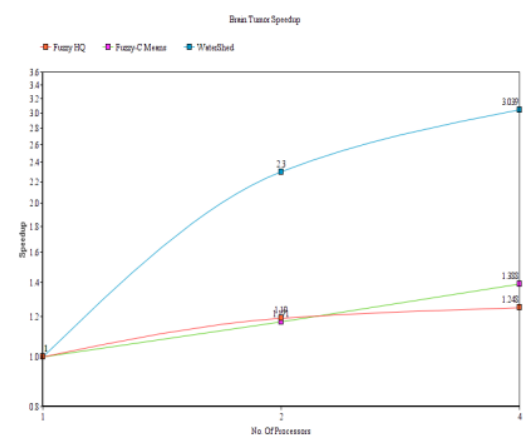

Figure 15. Speedup for Fuzzy-c means, Fuzzy-c means (Highquality) and watershed segmentation

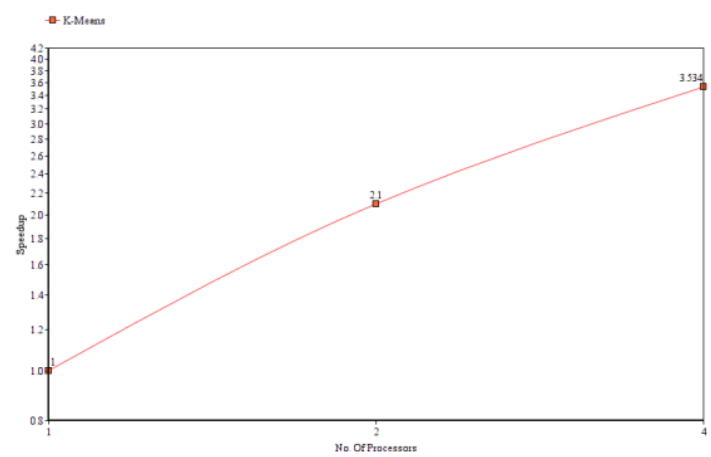

Figure 16. Speedup for K-means

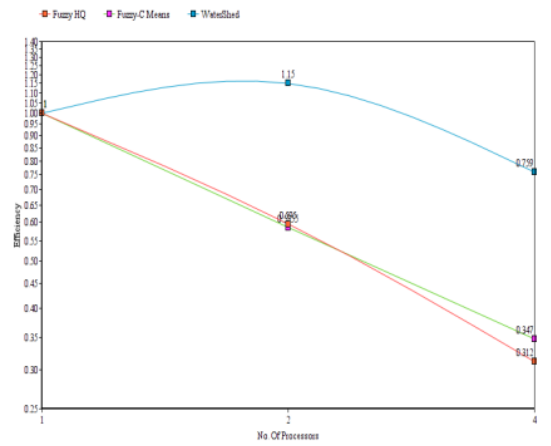

Figure 17. Efficiency for fuzzy-c means, fuzzy-c means (highQuality) and watershed segmentation

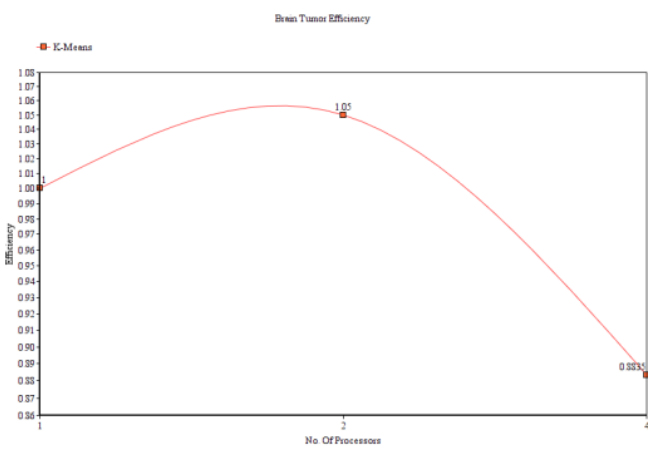

Figure 18. Efficiency for K-means

The above results (Ref. Table 1)are the time taken by the 3 algorithms. The implementation has been done in three stages sequential environment, parallel environment and implementation with a very high resolution image. The parallel execution as you takes lesser time to compute two images when sequential takes up almost the same amount of time for a single image this is achieved by creating parallel functions which enhances the performances of all the algorithms above thus making it a better way to detect tumors.

Fuzzy-c means showed an evident difference in performance with high resolution, to which our inference is that the algorithm allows multiple points to in multiple clusters but as the resolutions increases the pixels increases and hence number of points to cluster increase and this in turn increases the time taken to evaluate as it has to cluster many points in many clusters thus making the whole process complex and compute intensive.But when 2 images of high resolution were run in parallel the performance of Fuzzy-c means boosted to a significant level as mentioned in the Table 1.

We have further calculated speedup as well as efficiency of all the algorithms for up to 4 processors when executed in parallel. Where,

Speedup=Time(1)/Time $(p)$

i.e. time taken by 1 processor to execute the program by time taken by ' $p$ ' processors to execute the same program.

Efficiency $=$ Speedup/p

Where $p$ is number of processors.

After the calculations we have plotted graphs of speedup and efficiency for 1,2 and 4 processors as shown in figure $15-18$.

Form the graph we can see that speedup is noticed in each and everyalgorithm when executed in parallel environment. Watershed being one of themore efficient one and fcm being the most accurate. Here $\mathrm{K}$ means is showing high speedup but the case is algorithm is reducing the size of images along withlesser complexity as compared to fcm thus though higher speed but not muchefficient.

The efficiency graphs depict that there is a rise in the efficiency for 2 processors but as we increase processors the efficiency is reduced which is due to improper load balancing, background processes and system idle time but we can observe that the least reduction of efficiency is in watershed segmentation followed by fuzzy-c means and then $\mathrm{K}$-means which shows a big dip in efficiency as observed from the graphs. 


\section{Algorithm for Brain Tumor Detection}

\section{RESULT AND CONCLUSION}

In, this paper we have reviewed and implemented three of the known and famous algorithms for brain tumor detection and segmentation in a sequential as well as in a parallel environment. Our observations helps us to conclude that among the three algorithms watershed algorithm has an edge with the time efficiency and accuracy, thus when combined with the morphological image processing techniques it, gives us an edge over the orthodox clustering and segmentation algorithms for brain tumor detection as it also helps us in detecting the tumor as well as identifying which tumor sections are deeper in the brain as compared to other sections of the tumor. When it comes to accuracy when fuzzy-c mean along with the watershed algorithm is implemented, it possess higher accuracy but with a trade-off i.e. higher time consumption. Based on our observation we can also conclude that when these algorithms are implemented in a parallel environment their performance and efficiency increases drastically when it comes to evaluating multiple MRI's

\section{REFERENCES :}

1. Rastgarpour M., And Shanbehzadeh J., Application Of Ai Techniques In Medical Image Segmentation And Novel Categorization Of Available Methods And Tools, Proceedings Of The International Multiconference Of Engineers And Computer Scientists 2011 Vol I, Imecs 2011, March 16-18, 2011, Hongkong.

2. Zhang, Y. J, An Overview Of Image And Video Segmentation In The Last 40 Years, Proceedings Of The 6th International Symposium On Signal Processing And Its Applications, Pp. 144-151, 2001.

3. Wahba Marian, An Automated Modified Region Growing Technique For Prostate Segmentation In Trans-Rectal UltrasoundImages, Master"s Thesis, Department Of Electrical And Computer Engineering, University Of Waterloo, Waterloo, Ontario, Canada, 2008.

4. W. X. Kang, Q. Q. Yang, R. R. Liang,"The Comparative Research On Image Segmentation Algorithms", Ieee Conference On Etcs, Pp. 703-707, 2009.

5. N. R. Pal, S. K. Pal,“A Review On Image Segmentation Techniques”, Pattern Recognition, Vol. 26, No. 9,Pp.1277- 1294, 1993.

6. H. Zhang, J. E. Fritts, S. A. Goldman,"Image Segmentation Evaluation: A Survey Of Unsupervised Methods", Computer Vision And Image Understanding, Pp. 260-280, 2008.

7. L.Aurdal,"Image Segmentation Beyond Thresholding”, Norsk Regnescentral, 2006.

8. Y. Chang, X. Li, “Adaptive Image Region Growing”, Ieee Trans. On Image Processing, Vol. 3, No. 6, 1994.

9. K. Dehariya, S. K. Shrivastava, R. C. Jain, "Clustering Of Image Data Set Using K-Means And Fuzzy Kmeans Algorithms”, International Conference On Cicn, Pp.386- 391, 2010.

10. . Z. B. Chen, Q. H. Zheng, T. S. Qiu, Y. Liu. A New Method For Medical Ultrasonic Image Segmentation.Chinese Journal Of Biomedical Engineering.2006, 25(6),Pp.650-655.

11. A.Sivaramakrishnan And Dr.M.Karnan "A Novel Based Approach For Extraction Of Brain Tumor In Mri Images Using Soft Computing Techniques", International Journal Of Advanced Research In Computer And Communication Engineering, Vol. 2, Issue 4, April 2013.

12. K.Selvanayaki, Dr.P.Kalugasalam Intelligent Brain Tumor Tissue Segmentation From Magnetic Resonance Image (Mri) Using Meta Heuristic Algorithms Journal Of Global Research In Computer Science Volume 4, No. 2, February 2013

13. Kshitij Bhagwat, Dhanshri More, Sayali Shinde, Akshay Daga, Assistant Prof. Rupali Tornekar, " Comparative Study Of Brain Tumor Detection Using K Means ,Fuzzy C Means And Hierarchical Clustering Algorithms " International Journal Of Scientific \& Engineering Research, Volume 2,Issue 6,June 2013,Pp 626-632.

14. S.Roy And S.K.Bandoyopadhyay, "Detection And Qualification Of Brain Tumor From Mri Of Brain And Symmetric Analysis", International Journal Of Information And Communication Technology Research, Volume 2 No.6, June 2012, Pp584-588

15. . Tahir Sag Mehmet Cunkas, "Development Of Image Segmantation Techniques Using Swarm Intelligence”,Iccit 2012
16. Mohammed Sabbih Hamoud Al-Tamimi Ghazali Sulong, "Tumor Brain Detection Through Mr Images: A Review Of Literature”, Journal Of Theoretical And Applied Information Technology 20th April 2014. Vol. 62 No. 2

17. Sayali D. Gahukar et al Int. Journal of Engineering Research and Applications Vol. 4, Issue 4( Version 5), April 2014, pp.107-111.

18. . Shah, P., Jeshnani, M., Kukreja, S. and Ailani, P., 2017. Survey on algorithms for brain tumor detection. Int J Comput Sci Informat Technol, 8(1), pp.56-58.

19. Dhage, P., Phegade, M.R. and Shah, S.K., 2015, January. Watershed segmentation brain tumor detection. In 2015 International Conference on Pervasive Computing (ICPC) (pp. 1-5). IEEE.
Published By:

Blue Eyes Intelligence Engineering 